\title{
On the Entropy Rate of Pattern Processes
}

\author{
George M. Gemelos Tsachy Weissman \\ Department of Electrical Engineering \\ Stanford University \\ 350 Serra Mall \\ Stanford, CA 94305, USA \\ Email: \{ggemelos, tsachy\}@stanford.edu
}

October 28, 2018

\begin{abstract}
We study the entropy rate of pattern sequences of stochastic processes, and its relationship to the entropy rate of the original process. We give a complete characterization of this relationship for i.i.d. processes over arbitrary alphabets, stationary ergodic processes over discrete alphabets, and a broad family of stationary ergodic processes over uncountable alphabets. For cases where the entropy rate of the pattern process is infinite, we characterize the possible growth rate of the block entropy.
\end{abstract}

\section{Introduction}

In their recent work [8], Orlitsky et. al consider the compression of sequences with unknown alphabet size. This work, among others, has created interest in examining random processes with arbitrary alphabets which may a priori be unknown. One can think of this as a problem of reading a foreign language for the first time. As one begins to parse characters, one's knowledge of the alphabet grows. Since the characters in the alphabet have initially no meaning beyond the order in which they appear, one can relabel these characters by the order of their first appearance. Given a string, we refer to the relabelled string as the pattern associated with the original string.

Example 1 Assume that the following English sentence was being parsed into a pattern by a non-English speaker.

english is hard to learn... 
The associated pattern would be

$$
1,2,3,4,5,6,7,8,5,6,8,7,9,10,11,8,12,13,8,4,1,9,10,2, \ldots
$$

regarding the space too as a character.

We abstract this as follows: given a stochastic process $\mathbf{X}=\left\{X_{i}\right\}_{i \geq 1}$, we create a pattern process $\mathbf{Z}=\left\{Z_{i}\right\}_{i \geq 1}$.

It is the compression of the pattern process $\left\{Z_{i}\right\}$ that Orlitsky et. al focus on in [8]. This emphasis is justified by the fact that the bulk of the information is in the pattern. Although universal compression is an extensively studied problem, the universal compression of pattern sequences is relatively new, see [4, 5, 6, 7, 8, 10, 11, 12, 13, 14. The majority of these recent papers address universality questions of how well a pattern sequence associated with an unknown source can be compressed relative to the case where this distribution is known. Emphasis is on quantifying the redundancy, i.e., the difference between what can be achieved without and with knowledge of the source distribution. The main question we focus on in this work is how the entropy rate of a sequence and that of its pattern relate. More specifically, our goal is to study the relationship between the entropy rate $H(\mathbf{X})$ of the original process ${ }^{1}\left\{X_{i}\right\}$, and the entropy rate $H(\mathbf{Z})$ of the associated pattern process. This relationship is not always trivial, as the following examples illustrate.

Example 2 Let $X_{i}$ be drawn i.i.d. $\sim P$, where $P$ is a pmf on a finite alphabet. Then we show below that $H(\mathbf{X})=H(\mathbf{Z})$.

The intuition behind this result is that given enough time, all the symbols with positive probability will be seen, after which time the original process and its associated pattern sequence coincide, up to relabelling of the alphabet symbols.

Example 3 Let $X_{i}$ be drawn i.i.d. uniform $[0,1]$. Then the entropy rate of $\left\{X_{i}\right\}$ is $\infty$. Since the probability of seeing the same value twice is zero, $Z_{i}=i$ w.p. 1 for all $i$ and, consequently, $H(\mathbf{Z})=0$.

The connection between the entropy rate of the pattern and that of the original process was first studied for finite alphabet i.i.d. processes by Shamir and Song in [14]. The results in [14] gave bounds on the entropy rate of the pattern with respect to the entropy rate of the original process. These bounds were further extended to countably infinite alphabet i.i.d. processes in [12, 13]. These results were in the form of bounds, and did not completely characterize the relationship between the entropy rate of an i.i.d. process and that of its associated pattern. The first complete

\footnotetext{
${ }^{1}$ Throughout this work, $X_{m}^{n}$ will denote the sequence $X_{m}, X_{m+1}, \ldots, X_{n}$. If not specified, $m$ will be assumed to be 1 . Furthermore, $H(\mathbf{X})$ will denote entropy rate throughout this work, regardless of the discreteness of the distributions of $\left\{X^{n}\right\}$ (it should thus be regarded as $\infty$ when these are not discrete).
} 
characterization of this relationship for the general i.i.d. case as well as Markov, noise-corrupted and finite alphabet stationary ergodic processes, was given in [2] Orlitsky et. al in [7] independently derived the relationship for i.i.d. processes. The finite alphabet stationary ergodic result of [2] was later extended to general discrete stationary ergodic processes in [3], and independently in [9].

In this work we characterize the relationship between process and pattern entropy rates for general i.i.d., discrete Markov, and discrete stationary ergodic processes. Although the discrete Markov case falls under the more general results for discrete stationary ergodic sources, it will be seen that there is insight to be gained by exploring the discrete Markov case on its own. We then move on to examine stationary ergodic processes, with memory, over uncountable alphabets. In particular, we consider the Markov and additive noise case. These two results are then used to show a more general result for a broad family of stationary ergodic processes over uncountable alphabets. Finally, for the case where the entropy rate of the pattern process is infinite, we examine the possible growth rates for the block entropy of pattern processes.

In Section 2 we characterize the relationship between process and pattern entropy rate for the case of a generally distributed i.i.d. process. In Section [3, we examine the discrete Markov and the general discrete stationary ergodic process. Furthermore, in Section 4 we extend the uncountable alphabet results of Section 2 to certain processes with memory. In Section [5, we characterize a set of achievable asymptotic growth rates for the block entropy of a pattern process. We conclude in Section [6 with a brief summary of our results.

\section{The I.I.D. Case}

Consider the case where $X_{i}$ are generated i.i.d. $\sim f$, where $f$ is an arbitrary distribution on the arbitrary source alphabet $\mathcal{A}$. Let $S=\left\{x \in \mathcal{A}: \operatorname{Pr}\left\{X_{1}=x\right\}>0\right\}$.

Theorem 1 Given $X_{i}$ i.i.d. $\sim f$ and $\left\{Z_{i}\right\}$ its associated pattern process, for any point $x_{o} \notin S$ define the process

$$
\tilde{X}_{i}= \begin{cases}X_{i} & \text { if } X_{i} \in S \\ x_{o} & \text { otherwise }\end{cases}
$$

Then

$$
H(\mathbf{Z})=H(\tilde{\mathbf{X}})=H\left(\tilde{X}_{1}\right),
$$

regardless of the finiteness of both sides of the equality. ${ }^{2}$

\footnotetext{
${ }^{2}$ Throughout this work, we use $H$ to denote both entropy rate, when the argument is a process, and entropy, when the argument is a random variable.
} 
Since we will make use of a later corollary in the proof of Theorem 1, we present the proof in Appendix A. It should be noted that Theorem 1 was independently discovered by Orlitsky et al. in [7]. As can be seen, Theorem 11 is consistent with Example 2 and Example 3. Note that the process $\left\{\tilde{X}_{i}\right\}$ is created by keeping all the point masses in $S$ and assigning all the remaining probability to a new point mass. This corresponds with the result in Example 3 which suggests that the pattern of a process drawn according to a pdf has no randomness, i.e. an entropy rate of zero. Therefore, the only randomness in the pattern comes from the point masses and the event of falling on a "non-point-mass-mode".

Example 4 Let $\left\{X_{i}\right\}$ be an i.i.d. process with each component drawn, with probability $1 / 3$, as $N(0,1)$ and, with probability $2 / 3$, as Bernoulli $1 / 2$. In this case $\tilde{X}_{i}$ is uniformly distributed on an alphabet of size 3. Therefore, Theorem 1 gives $H(\mathbf{Z})=\log (3)$.

Although $|S|<\infty$ in all three examples above, it should be noted that Theorem 1 makes no such assumption.

\section{Discrete Alphabet Processes}

Having characterized the relationship between process and pattern entropy rate for the general i.i.d. process, what can be said about processes with memory? To begin exploring the answer to this question we examine one of the most basic stationary ergodic processes with memory, the Markov process.

\section{A Markov Processes over Discrete Alphabets}

Although discrete Markov processes fall under the more general Theorem 3 to follow, which deals with discrete stationary ergodic processes, there is insight gained by examining the Markov case on it own. In particular, we will see that the proof of the general discrete stationary ergodic result relies heavily on a version of the ShannonMcMillan-Breiman theorem for countably infinite alphabets, found in [1], while no such heavy machinery is necessary for the simpler Markov case. This fact is due to the inherent structure of a Markov process and makes the Markov case an interesting example on it own. Later on in Section 4, we will also see it is this structure which makes the Markov process the first candidate for the extension of the uncountable alphabet results of Section 2 to uncountable alphabet processes with memory.

The entropy rate of Markov processes is well-known. What can be said about the entropy rate of the associated pattern processes? We first look at the case of a first order Markov process with components in a countable alphabet.

Proposition 1 Let $\left\{X_{i}\right\}$ be a stationary ergodic first order Markov process on the countable alphabet $\mathcal{A}$ and let $\left\{Z_{i}\right\}$ be the associated pattern process. If $H(\mathbf{X})<\infty$, 
then

$$
H(\mathbf{Z})=H(\mathbf{X}) .
$$

Proof of Proposition [1;

Let $\mu$ be the stationary distribution of the Markov process and let $P_{x}(y)=P\left(X_{t+1}=\right.$ $\left.y \mid X_{t}=x\right)$ for all $x, y \in \mathcal{A}$. The data processing inequality implies $H\left(X^{n}\right) \geq H\left(Z^{n}\right)$ for all $n$. Hence $H(\mathbf{X}) \geq H(\mathbf{Z})$. To complete the proof it remains to show $H(\mathbf{X}) \leq H(\mathbf{Z})$, for which we will need the following two lemmas.

Lemma 1 Let $\left\{A_{n}\right\}$ and $\left\{B_{n}\right\}$ be two sequences of events such that $\lim _{n \rightarrow \infty} P\left(A_{n}\right)=$ 1 and $\lim _{n \rightarrow \infty} P\left(B_{n}\right)=b$. Then $\lim _{n \rightarrow \infty} P\left(A_{n} \cap B_{n}\right)=b$.

Proof of Lemma 团:

$P\left(A_{n} \cap B_{n}\right) \leq P\left(B_{n}\right) \rightarrow b$. On the other hand,

$$
\begin{aligned}
\liminf _{n \rightarrow \infty} P\left(A_{n} \cap B_{n}\right) & =\liminf _{n \rightarrow \infty} 1-P\left(A_{n}^{c} \cup B_{n}^{c}\right) \\
& \geq \liminf _{n \rightarrow \infty} 1-P\left(A_{n}^{c}\right)-P\left(B_{n}^{c}\right) \\
& =1-0-(1-b) .
\end{aligned}
$$

Lemma 2 Given any $B \subseteq \mathcal{A}$ such that $|B|<\infty$

$$
H(\mathbf{Z}) \geq \sum_{b \in B} \mu(b) H\left(\Phi_{B}\left[P_{b}\right]\right),
$$

where we define the pmf $\Phi_{B}\left[P_{b}\right](x)=1_{B}(x) P_{b}(x)+P_{b}\left(B^{c}\right) \delta_{x_{o}}(x)$, for an arbitrary $x_{o} \notin B .{ }^{3}$ Here $\delta_{x_{o}}$ is used to denote the distribution which places unit mass on $x_{o}$.

Proof of Lemma 娄:

Let $A\left(X^{n}\right)$ be the set of distinct elements in $\left\{X_{1}, \ldots, X_{n}\right\}$. Then

$$
\begin{aligned}
H(\mathbf{Z}) & =\lim _{n \rightarrow \infty} \frac{H\left(Z^{n}\right)}{n} \\
& \stackrel{(a)}{=} \lim _{n \rightarrow \infty} H\left(Z_{n} \mid Z^{n-1}\right) \\
& \geq \lim _{n \rightarrow \infty} H\left(Z_{n} \mid X^{n-1}\right) \\
& \geq \lim _{n \rightarrow \infty} \sum_{b \in B} \int_{\left\{x^{n-1}: B \subseteq A\left(x^{n-1}\right), x_{n-1}=b\right\}} H\left(P\left(Z_{n} \mid X^{n-1}=x^{n-1}\right)\right) d P_{X^{n-1}}
\end{aligned}
$$

\footnotetext{
${ }^{3}$ Throughout this work, given a distribution $f$ and a set $B, \Phi_{B}[f]$ will denote the distribution defined by $\Phi_{B}[f](x)=1_{B}(x) f(x)+f\left(B^{c}\right) \delta_{x_{o}}(x)$ for an arbitrary $x_{o} \notin B$. When $f$ is a distribution, $H(f)$ will denote the entropy of a random variable drawn according to $f$. Furthermore, $1_{A}$ will denote the indicator function on the set $A$, while $\mathbb{1}_{A}$ will denote the indicator random variable on the event $A$.
} 


$$
\begin{aligned}
& \geq \lim _{n \rightarrow \infty} \sum_{b \in B} P\left(B \subseteq A\left(X^{n-1}\right), X_{n-1}=b\right) H\left(\Phi_{B}\left[P_{b}\right]\right) \\
& =\sum_{b \in B} H\left(\Phi_{B}\left[P_{b}\right]\right) \lim _{n \rightarrow \infty} P\left(B \subseteq A\left(X^{n-1}\right), X_{n-1}=b\right) \\
& \geq \sum_{b \in B} \mu(b) H\left(\Phi_{B}\left[P_{b}\right]\right)
\end{aligned}
$$

where $(a)$ follows from the fact that $H\left(Z_{1}\right)<\infty$ and $H\left(Z_{n} \mid Z^{n-1}\right)<\infty$ for all $n$ and (b) from Lemma 11 since, $\operatorname{Pr}\left\{B \subseteq A\left(X^{n}\right)\right\} \rightarrow 1$.

Now let $\left\{B_{k}\right\}$ be a sequence of sets such that $B_{k} \subseteq \mathcal{A},\left|B_{k}\right|<\infty$ for all $k$, and

$$
\lim _{k \rightarrow \infty} \sum_{b \in B_{k}} \sum_{a \in B_{k}}-\mu(b) P_{b}(a) \log P_{b}(a)=\sum_{b \in \mathcal{A}} \sum_{a \in \mathcal{A}}-\mu(b) P_{b}(a) \log P_{b}(a)
$$

regardless of the finiteness of both sides of the equation. Note that since the above summands are all positive, such a sequence $\left\{B_{k}\right\}$ can always be found. Lemma 2 gives

$$
H(\mathbf{Z}) \geq \sum_{b \in B_{k}} \mu(b) H\left(\Phi_{B_{k}}\left[P_{b}\right]\right) \quad \forall k .
$$

Hence, by taking $k \rightarrow \infty$, we get

$$
\begin{aligned}
H(\mathbf{Z}) & \geq \lim _{k \rightarrow \infty} \sum_{b \in B_{k}} \mu(b) H\left(\Phi_{B_{k}}\left[P_{b}\right]\right) \\
& \geq \lim _{k \rightarrow \infty} \sum_{b \in B_{k}} \mu(b) \sum_{a \in B_{k}}-P_{b}(a) \log P_{b}(a) \\
& =\lim _{k \rightarrow \infty} \sum_{b \in B_{k}} \sum_{a \in B_{k}}-\mu(b) P_{b}(a) \log P_{b}(a) \\
& \stackrel{(a)}{=} \sum_{b \in \mathcal{A}} \sum_{a \in \mathcal{A}}-\mu(b) P_{b}(a) \log P_{b}(a) \\
& \stackrel{(b)}{=} H(\mathbf{X})
\end{aligned}
$$

where $(a)$ comes from the construction of $\left\{B_{k}\right\}$ and $(b)$ from the fact that $\left\{X_{i}\right\}$ is a finite entropy first order Markov process.

One should note that the proof of Proposition 1 can easily be extended to the case of Markov processes of any order. Hence, without going through the proof, we state the following:

Theorem 2 Let $\left\{X_{i}\right\}$ be a stationary ergodic Markov process of order $m$ on the countable alphabet $\mathcal{A}$, and let $\left\{Z_{i}\right\}$ be the associated pattern process. If $H(\mathbf{X})<\infty$, then

$$
H(\mathbf{Z})=H(\mathbf{X})
$$




\section{B Stationary Ergodic Processes over Discrete Alphabets}

Now that we have characterized the entropy rate relationship for the discrete Markov process, the natural next step would be to extend the results to all stationary ergodic processes on a countable alphabet.

Theorem 3 Let $\left\{X_{i}\right\}$ be a stationary ergodic process with components taking values from the countable alphabet $\mathcal{A}$, and assume $H(\mathbf{X})<\infty$. Let $\left\{Z_{i}\right\}$ be the associated pattern process. Then

$$
H(\mathbf{Z})=H(\mathbf{X})
$$

We will see that as compared to the proof of Proposition 1, the structure of the proof of Theorem [3 is slightly different, using a sandwich argument, and making use of heavier machinery such as a version of the Shannon-McMillan-Breiman theorem for countably infinite alphabets [1].

It is also important to note that like Theorem 2, Theorem 3 also has a finite entropy constraint. The need to exclude processes with infinite entropy from Theorem 3 is a direct result of the requirement of finite entropy for the countably infinite version of the Shannon-McMillan-Breiman theorem.

The proof of Theorem 3 will use the following two claims.

Claim 1 Let $Z_{0}^{(n)}$ be the pattern element at time zero after seeing $\left(X_{-1}, \ldots, X_{-n}\right)$.

$$
\lim _{n \rightarrow \infty} H\left(Z_{0}^{(n)} \mid X_{-n}^{-1}\right)=H\left(X_{0} \mid X_{-\infty}^{-1}\right)
$$

Proof of Claim [1

It is sufficient to show

$$
\lim _{n \rightarrow \infty} H\left(X_{0} \mid X_{-n}^{-1}\right)=H\left(X_{0} \mid X_{-\infty}^{-1}\right)
$$

and

$$
\lim _{n \rightarrow \infty}\left|H\left(X_{0} \mid X_{-n}^{-1}\right)-H\left(Z_{0}^{(n)} \mid X_{-n}^{-1}\right)\right|=0 .
$$

From [1] we know that $-\log \left(P\left(X_{0} \mid X_{-n}^{-1}\right)\right) \rightarrow-\log \left(P\left(X_{0} \mid X_{-\infty}^{-1}\right)\right)$ a.s. and the sequence is uniformly integrable, implying (11).

Moving on to (2) we see that the data processing inequality gives us $H\left(X_{0} \mid X_{-n}^{-1}\right) \geq$ $H\left(Z_{0}^{(n)} \mid X_{-n}^{-1}\right)$ for all $n$. Hence it will suffice to show

$$
\limsup _{n \rightarrow \infty} H\left(X_{0} \mid X_{-n}^{-1}\right)-H\left(Z_{0}^{(n)} \mid X_{-n}^{-1}\right) \leq 0
$$


Let $A\left(X_{-n}^{-1}\right)$ be the set of distinct elements in $\left\{X_{-1}, \ldots, X_{-n}\right\}$. Then

$$
\begin{aligned}
& H\left(X_{0} \mid X_{-n}^{-1}\right)-H\left(Z_{0}^{(n)} \mid X_{-n}^{-1}\right)=\mathbb{E}\left[\sum_{x \in \mathcal{A}} P_{X_{0} \mid X_{-n}^{-1}}(x) \log \left(\frac{1}{P_{X_{0} \mid X_{-n}^{-1}}(x)}\right)\right] \\
& -\mathbb{E}\left[\sum_{x \in A\left(X_{-n}^{-1}\right)} P_{X_{0} \mid X_{-n}^{-1}}(x) \log \left(\frac{1}{P_{X_{0} \mid X_{-n}^{-1}}(x)}\right)\right] \\
& -\mathbb{E}\left[P_{X_{0} \mid X_{-n}^{-1}}\left(A\left(X_{-n}^{-1}\right)^{c}\right) \log \left(\frac{1}{P_{X_{0} \mid X_{-n}^{-1}}\left(A\left(X_{-n}^{-1}\right)^{c}\right)}\right)\right] \\
& =\mathbb{E}\left[\sum_{x \in A\left(X_{-n}^{-1}\right)^{c}} P_{X_{0} \mid X_{-n}^{-1}}(x) \log \left(\frac{1}{P_{X_{0} \mid X_{-n}^{-1}}(x)}\right)\right] \\
& -\mathbb{E}\left[P_{X_{0} \mid X_{-n}^{-1}}\left(A\left(X_{-n}^{-1}\right)^{c}\right) \log \left(\frac{1}{P_{X_{0} \mid X_{-n}^{-1}}\left(A\left(X_{-n}^{-1}\right)^{c}\right)}\right)\right] \\
& \leq \mathbb{E}\left[\sum_{x \in A\left(X_{-n}^{-1}\right)^{c}} P_{X_{0} \mid X_{-n}^{-1}}(x) \log \left(\frac{1}{P_{X_{0} \mid X_{-n}^{-1}}(x)}\right)\right] \text {. }
\end{aligned}
$$

Since $H\left(X_{0}\right)<\infty$, given $\epsilon>0$ there exists a $B \subset \mathcal{A}$ such that: $|B|<\infty$, if $b \in B$ then $\operatorname{Pr}\left\{X_{0}=b\right\}>0$, and

$$
H\left(\Phi_{B^{c}}\left[P_{X}\right]\right) \leq \epsilon
$$

where $P_{X}$ is the distribution on $X_{0}$ and $\Phi_{B^{c}}\left[P_{X}\right]$ is defined as in Lemma 2 . Since

$$
\mathbb{E}\left[\sum_{x \in B^{c}} P_{X_{0} \mid X_{-n}^{-1}}(x) \log \left(\frac{1}{P_{X_{0} \mid X_{-n}^{-1}}(x)}\right)\right] \leq H\left(\Phi_{B^{c}}\left[P_{\mathbf{X}}\right] \mid X_{-n}^{-1}\right) \leq H\left(\Phi_{B^{c}}\left[P_{\mathbf{X}}\right]\right) \quad \forall n
$$

(41) implies

$$
\mathbb{E}\left[\sum_{x \in B^{c}} P_{X_{0} \mid X_{-n}^{-1}}(x) \log \left(\frac{1}{P_{X_{0} \mid X_{-n}^{-1}}(x)}\right)\right] \leq \epsilon \quad \forall n .
$$

By the ergodicity of $\left\{X_{i}\right\}$

$$
\lim _{n \rightarrow \infty} \operatorname{Pr}\left\{B \subset A\left(X_{-n}^{-1}\right)\right\}=1 .
$$


From (3) and the construction of $B$ we get

$$
\begin{aligned}
& H\left(X_{0} \mid X_{-n}^{-1}\right)-H\left(Z_{0}^{(n)} \mid X_{-n}^{-1}\right) \leq \mathbb{E}\left[\mathbb{1}_{\left\{B \subset A\left(X_{-n}^{-1}\right)\right\}} \sum_{x \in A\left(X_{-n}^{-1}\right)^{c}} P_{X_{0} \mid X_{-n}^{-1}}(x) \log \left(\frac{1}{P_{X_{0} \mid X_{-n}^{-1}}(x)}\right)\right] \\
& +\mathbb{E}\left[\mathbb{1}_{\left\{B \not \subset A\left(X_{-n}^{-1}\right)\right\}} \sum_{x \in A\left(X_{-n}^{-1}\right)^{c}} P_{X_{0} \mid X_{-n}^{-1}}(x) \log \left(\frac{1}{P_{X_{0} \mid X_{-n}^{-1}}(x)}\right)\right] \\
& \leq \mathbb{E}\left[\mathbb{1}_{\left\{B \subset A\left(X_{-n}^{-1}\right)\right\}} \sum_{x \in B^{c}} P_{X_{0} \mid X_{-n}^{-1}}(x) \log \left(\frac{1}{P_{X_{0} \mid X_{-n}^{-1}}(x)}\right)\right] \\
& +H\left(X_{0} \mid X_{-n}^{-1}\right) \mathbb{E}\left[\mathbb{1}_{\left\{B \not \subset A\left(X_{-n}^{-1}\right)\right\}}\right] \\
& \leq \mathbb{E}\left[\mathbb{1}_{\left\{B \subset A\left(X_{-n}^{-1}\right)\right\}} \sum_{x \in B^{c}} P_{X_{0} \mid X_{-n}^{-1}}(x) \log \left(\frac{1}{P_{X_{0} \mid X_{-n}^{-1}}(x)}\right)\right] \\
& +H\left(X_{0}\right) \mathbb{E}\left[\mathbb{1}_{\left\{B \not \subset A\left(X_{-n}^{-1}\right)\right\}}\right] \\
& \leq \epsilon \mathbb{E}\left[\mathbb{1}_{\left\{B \subset A\left(X_{-n}^{-1}\right)\right\}}\right]+H\left(X_{0}\right) \mathbb{E}\left[\mathbb{1}_{\left\{B \not \subset A\left(X_{-n}^{-1}\right)\right\}}\right] \\
& \leq \epsilon \operatorname{Pr}\left\{B \subset A\left(X_{-n}^{-1}\right)\right\}+H\left(X_{0}\right)\left(1-\operatorname{Pr}\left\{B \subset A\left(X_{-n}^{-1}\right)\right\}\right) \text {. }
\end{aligned}
$$

Taking the limit in $n$, (15) gives

$$
\limsup _{n \rightarrow \infty} H\left(X_{0} \mid X_{-n}^{-1}\right)-H\left(Z_{0}^{(n)} \mid X_{-n}^{-1}\right) \leq \epsilon .
$$

Since $\epsilon$ was arbitrary, (2) follows, completing the proof of Claim 10.

\section{Claim 2}

$$
H(\mathbf{X})=H\left(X_{0} \mid X_{-\infty}^{-1}\right) .
$$

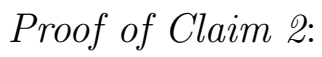

From [1] we know that $-\log \left(P\left(X_{0} \mid X_{-n}^{-1}\right)\right) \rightarrow-\log \left(P\left(X_{0} \mid X_{-\infty}^{-1}\right)\right)$ a.s. and the sequence is uniformly integrable. Therefore, uniform intergrability and almost sure convergence implies convergence in mean.

We are now ready for:

Proof of Theorem ;

$$
\begin{aligned}
H(\mathbf{X}) & =\limsup _{n \rightarrow \infty} \frac{1}{n} H\left(X^{n}\right) \\
& \stackrel{(a)}{\geq} \limsup _{n \rightarrow \infty} \frac{1}{n} H\left(Z^{n}\right)
\end{aligned}
$$




$$
\begin{aligned}
& \geq \liminf _{n \rightarrow \infty} \frac{1}{n} H\left(Z^{n}\right) \\
& =\liminf _{n \rightarrow \infty} \frac{1}{n} \sum_{i=0}^{n-1} H\left(Z_{i+1} \mid Z^{i}\right) \\
& \geq \liminf _{n \rightarrow \infty} H\left(Z_{n+1} \mid Z^{n}\right) \\
& \stackrel{(b)}{=} \liminf _{n \rightarrow \infty} H\left(Z_{0}^{(n)} \mid Z_{-n}^{-1}\right) \\
& \geq \liminf _{n \rightarrow \infty} H\left(Z_{0}^{(n)} \mid X_{-n}^{-1}\right) \\
& \stackrel{(c)}{=} H\left(X_{0}^{(n)} \mid X_{-\infty}^{-1}\right) \\
& \stackrel{(d)}{=} H(\mathbf{X})
\end{aligned}
$$

where $(a)$ follows from the data processing inequality, (b) is a result of stationarity, (c) results from Claim 1 and $(d)$ results from Claim 2.

\section{Uncountable Alphabet Processes with Memory}

The i.i.d. results of Theorem 1 completely characterize the entropy rate relationship for the general memoryless stationary process. So far, we have only addressed the case of discrete processes with memory. A natural question that arises is whether the relationship between the entropy rate of the process and that of the pattern shown in Theorem 1 can be extended to processes with memory over an uncountable alphabet? Once again, we first look at Markov processes.

\section{A Markov Processes over Uncountable Alphabets}

We observed in Section 3 that the inherent structure of the Markov process simplified the proof of the results in the discrete case. The hope is that by looking at this heavily structured family first we will develop some insight into the more general case of a stationary ergodic process over an uncountable alphabet.

Although we are unable to characterize the entropy rate of the induced pattern process for a general uncountable alphabet Markov process, the following proposition covers a fairly general family of Markov processes. Before we state the proposition, let us generalize some of the notation used in Proposition 1, Given an $m^{\text {th }}$ order Markov process $\left\{X_{i}\right\}$ on $\mathbb{R}$, for $x^{m} \in \mathbb{R}^{m}$ let $f_{x^{m}}$ be the kernel associated with the state $x^{m}$. We will denote the set of point masses of $f_{x^{m}}$ as $S_{x^{m}}, S_{x^{m}}=\left\{y \in \mathbb{R}: \operatorname{Pr}\left\{X_{m+1}=\right.\right.$ $\left.\left.y \mid X^{m}=x^{m}\right\}>0\right\}$.

Proposition 2 Let $\left\{X_{i}\right\}$ be a stationary ergodic Markov process on $\mathbb{R}$ of order $m$ such that there exists $S \subset \mathbb{R}$ with $S_{x^{m}}=S$ for all $x^{m} \in \mathbb{R}^{m}$ and $\Phi_{S}\left[f_{x^{m}}\right]=\Phi_{S}\left[f_{y^{m}}\right]$ 
for all $x^{m}, y^{m} \notin S^{m}$. Let $\left\{Z_{i}\right\}$ be the pattern process associated with $\left\{X_{i}\right\}$. Define the process $\left\{\tilde{X}_{i}\right\}$ as

$$
\tilde{X}_{i}= \begin{cases}X_{i} & \text { if } X_{i} \in S \\ x_{o} & \text { otherwise }\end{cases}
$$

for an arbitrary $x_{o} \in S^{c}$. If $|S|<\infty$, then

$$
H(\mathbf{Z})=H(\tilde{\mathbf{X}})
$$

The proof of Proposition 2, as well as the remaining results of the present section begins with the observation that Theorem 3 implies $H(\tilde{\mathbf{X}})=H(\tilde{\mathbf{Z}})$. It is then left to show that $H(\mathbf{Z})$ is equal to $H(\tilde{\mathbf{Z}})$. To this end, we show that for any given $n$, the difference between $H\left(Z^{n}\right)$ and $H\left(\tilde{Z}^{n}\right)$ is either bounded or grows sub-linearly in $n$.

Proof of Proposition 2:

If $|S|=0$, w.p. 1 the process $\left\{X_{i}\right\}$ does not repeat and therefore $H(\mathbf{Z})=0$. Similarly if $|S|=0$, the process $\left\{\tilde{X}_{i}\right\}$ is a constant and therefore $H(\tilde{\mathbf{X}})=0$. Hence $H(\mathbf{Z})=H(\tilde{\mathbf{X}})$.

We now look at the case where $|S|>0$. We observe that since $\Phi_{S}\left[f_{x^{m}}\right]=\Phi_{S}\left[f_{y^{m}}\right]$ for all $x^{m}, y^{m} \notin S^{m},\left\{\tilde{X}_{i}\right\}$ is a discrete Markov process of order $m$. Hence Theorem 2 and the fact that $\left\{\tilde{X}_{i}\right\}$ is stationary ergodic and has finite entropy gives

$$
H(\tilde{\mathbf{X}})=H(\tilde{\mathbf{Z}})
$$

where $\left\{\tilde{Z}_{i}\right\}$ is the pattern process associated with $\left\{\tilde{X}_{i}\right\}$.

For $x \in S$ define the waiting time $I_{x}=\inf \left\{i>0: X_{i}=x\right\}$. Given $\left\{I_{x}\right\}_{x \in S}$ we know the first appearance of every point in $S$. Hence we know the first appearance of every point but those which are assigned zero probability by every kernel, i.e. all but those that appear at most once w.p. 1. Therefore given $\tilde{Z}^{n}$ and $\left\{I_{x}\right\}_{x \in S}$ we can reconstruct $Z^{n}$ w.p. 1 for all $n$. Similarly given $Z^{n}$ and $\left\{I_{x}\right\}_{x \in S}$ we can reconstruct $\tilde{Z}^{n}$ for all $n$. Hence

$$
H\left(Z^{n}\right) \leq H\left(\tilde{Z}^{n}\right)+\sum_{x \in S} H\left(I_{x}\right) \quad \forall n
$$

and

$$
H\left(Z^{n}\right)+\sum_{x \in S} H\left(I_{x}\right) \geq H\left(\tilde{Z}^{n}\right) \quad \forall n .
$$

\section{Claim 3}

$$
H\left(I_{x}\right)<\infty \quad \forall x \in S
$$

Proof of Claim 3 ;

Given $x \in S$, define $d_{\text {min }}=\min \left\{\operatorname{Pr}\left\{X_{m+1}=x \mid X^{m}=y^{m}\right\}: y^{m} \in \mathbb{R}^{m}\right\}$ and $d_{\max }=$ $\max \left\{\operatorname{Pr}\left\{X_{m+1}=x \mid X^{m}=y^{m}\right\}: y^{m} \in \mathbb{R}^{m}\right\}$. Note that since $0<|S|<\infty$ and 
$\Phi_{S}\left[f_{x^{m}}\right]=\Phi_{S}\left[f_{y^{m}}\right]$ for all $x^{m}, y^{m} \notin S^{m}, d_{\text {min }}$ and $d_{\max }$ are well defined. By the definition of $S, d_{\min }>0$.

First, let us consider the case where $d_{\max }=1$. Then, there exists a $x \in S$ and $y^{m} \in \mathbb{R}^{m}$ such that $\operatorname{Pr}\left\{X_{m+1}=x \mid X^{m}=y^{m}\right\}=1$ and $S=S_{y^{m}}=\{x\}$. We will first look at the case where $y^{m} \in S^{m}$. Since $S=\{x\}$, if $y^{m} \in S^{m}$, then $y^{m}=x^{m}$, where $x^{m}$ is the vector $(x, \ldots, x)$ of length $m$. Therefore $f_{x^{m}}=\delta_{x}$ and once the state $x^{m}$ is reached it cannot be exited. Hence in order for $\left\{\tilde{X}_{i}\right\}$ to be irreducible, which is required for the process to be ergodic, it must place zero or unit probability on being in state $x^{m}$. By the construction of $S$ and the fact that $x \in S, \operatorname{Pr}\left\{X^{m}=x^{m}\right\}>0$ and therefore $\operatorname{Pr}\left\{\tilde{X}^{m}=x^{m}\right\}>0$. Hence $X_{i}=x$ w.p. 1 and $H\left(I_{x}\right)=0$. Let us now examine the case where $y^{m} \notin S^{m}$. Since $\Phi_{S}\left[f_{u^{m}}\right]=\Phi_{S}\left[f_{y^{m}}\right]$ for all $u^{m}, y^{m} \notin S^{m}$, if $u^{m} \notin S^{m}$, then $\operatorname{Pr}\left\{X_{m+1}=x \mid X^{m}=u^{m}\right\}=1$. Noting that $S=\{x\}$, we can conclude that if $X_{i} \neq x$, then w.p. $1 X_{i+1}=x$. Hence $I_{x} \leq 2$ and $H\left(I_{x}\right) \leq \log 2$.

We now consider the less trivial case where $d_{\max }<1$. Since regardless of the state $y^{m}, \operatorname{Pr}\left\{X_{m+1}=x \mid X^{m}=y^{m}\right\} \in\left[d_{\min }, d_{\max }\right]$, then

$$
\operatorname{Pr}\left\{I_{x}=i\right\} \in\left[d_{\min }\left(1-d_{\max }\right)^{i-1}, d_{\max }\left(1-d_{\text {min }}\right)^{i-1}\right] \quad \forall i \in \mathbb{N} .
$$

Hence

$$
\begin{aligned}
H\left(I_{x}\right)= & \sum_{i=1}^{\infty} \operatorname{Pr}\left\{I_{x}=i\right\} \log \left(\frac{1}{\operatorname{Pr}\left\{I_{x}=i\right\}}\right) \\
\leq & \sum_{i=1}^{\infty} d_{\max }\left(1-d_{\min }\right)^{i-1} \log \left(\frac{1}{d_{\min }\left(1-d_{\max }\right)^{i-1}}\right) \\
= & -d_{\max } \sum_{i=1}^{\infty}\left(1-d_{\min }\right)^{i-1}\left[\log \left(d_{\min }\right)+\log \left(\left(1-d_{\max }\right)^{i-1}\right)\right] \\
= & -d_{\max } \sum_{i=1}^{\infty}\left(1-d_{\min }\right)^{i-1} \log \left(d_{\min }\right) \\
& -d_{\max } \sum_{i=1}^{\infty}(i-1)\left(1-d_{\min }\right)^{i-1} \log \left(1-d_{\max }\right) \\
= & -d_{\max } \log \left(d_{\min }\right) \sum_{i=0}^{\infty}\left(1-d_{\min }\right)^{i} \\
& -d_{\max } \log \left(1-d_{\max }\right) \sum_{i=0}^{\infty} i\left(1-d_{\min }\right)^{i} \\
= & -\frac{d_{\max } \log \left(d_{\min }\right)}{d_{\min }}-\frac{d_{\max }\left(1-d_{\min }\right) \log \left(1-d_{\max }\right)}{d_{\min }^{2}} \\
&
\end{aligned}
$$

Since $d_{\min }, d_{\max } \in(0,1)$, equation (9) implies $H\left(I_{x}\right)<\infty$.

Claim 3 therefore gives

$$
\lim _{n \rightarrow \infty} \frac{H\left(I_{x}\right)}{n}=0 \quad \forall n \in \mathbb{N}, x \in S .
$$


Combining equations (17), (8), (10) and noting that $|S|<\infty$ gives $H(\mathbf{Z})=H(\tilde{\mathbf{Z}})$. Equation (6) then completes the proof.

Example 5 Let $\left\{X_{i}\right\}$ be a first order Markov process on $[0,1]$ with the following transition kernels, represented as generalized densities on $[0,1]$ :

$$
\begin{aligned}
& f_{0}(y)=\frac{3}{4} \delta_{0}(y)+\frac{1}{4} \delta_{1}(y) \\
& f_{1}(y)=\frac{1}{4} \delta_{0}(y)+\frac{1}{2} \delta_{1}(y)+\frac{1}{4},
\end{aligned}
$$

and for $x \in(0,1)$

$$
\begin{aligned}
f_{x}(y) & =\frac{1}{4} \delta_{0}(y)+\frac{1}{4} \delta_{1}(y)+\frac{3}{4} 1_{\{(x-1 / 2)(y-1 / 2)>0\}}(y) \\
& +\frac{1}{4} 1_{\{(x-1 / 2)(y-1 / 2) \leq 0\}}(y) .
\end{aligned}
$$

It is readily checked that the stationary distribution given the above kernels is

$$
\mu(x)=\frac{1}{2} \delta_{0}(x)+\frac{1}{3} \delta_{1}(x)+\frac{1}{6} \quad \forall x \in[0,1] .
$$

In the above case, $\left\{\tilde{X}_{i}\right\}$ can be thought of as a first order Markov process on the set $\{0,1 / 2,1\}$, (the value $1 / 2$ chosen arbitrarily) with transition probabilities whose generalized densities are

$$
\begin{aligned}
\tilde{f}_{0}(y) & =\frac{3}{4} \delta_{0}(y)+\frac{1}{4} \delta_{1}(y) \\
\tilde{f}_{1}(y) & =\frac{1}{4} \delta_{0}(y)+\frac{1}{2} \delta_{1}(y)+\frac{1}{4} \delta_{1 / 2}(y) \\
\tilde{f}_{1 / 2}(y) & =\frac{1}{4} \delta_{0}(y)+\frac{1}{4} \delta_{1}(y)+\frac{1}{2} \delta_{1 / 2}(y) .
\end{aligned}
$$

Hence $\left\{\tilde{X}_{i}\right\}$ has the following stationary distribution

$$
\tilde{\mu}(x)=\frac{1}{2} \delta_{0}(x)+\frac{1}{3} \delta_{1}(x)+\frac{1}{6} \delta_{1 / 2}(x) .
$$

Applying Proposition 2 gives

$$
\begin{aligned}
H(\mathbf{Z}) & =H(\tilde{\mathbf{X}}) \\
& =\frac{1}{2} H\left(\tilde{X}_{2} \mid \tilde{X}_{1}=0\right)+\frac{1}{3} H\left(\tilde{X}_{2} \mid \tilde{X}_{1}=1\right)+\frac{1}{6} H\left(\tilde{X}_{2} \mid \tilde{X}_{1}=1 / 2\right) \\
& =\frac{1}{2}\left(2-\frac{3}{4} \log 3\right)+\frac{1}{3}\left(\frac{3}{2}\right)+\frac{1}{6}\left(\frac{3}{2}\right) \\
& =\frac{7}{4}-\frac{3}{8} \log (3)=1.1556 .
\end{aligned}
$$




\section{B Additive White Noise-Corrupted Processes}

We now consider the case of a noise-corrupted process. Let $\left\{X_{i}\right\}$ be a stationary ergodic process and $\left\{Y_{i}\right\}$ be its noise-corrupted version. Here we assume i.i.d. additive noise, $\left\{N_{i}\right\}$, with $X_{i}, Y_{i}$, and $N_{i}$ taking values in $\mathbb{R}$. Let $S_{Y}$ and $S_{N}$ denote the set of point masses for $Y_{i}$ and $N_{i}$ respectively. We will also define the process

$$
\tilde{N}_{i}=\left\{\begin{array}{cc}
N_{i} & \text { if } N_{i} \in S_{N} \\
n_{o}-X_{i} & \text { otherwise }
\end{array}\right.
$$

for an arbitrary point $n_{o} \notin S_{Y}$.

Proposition 3 Let $\left\{X_{i}\right\}$ be a finite alphabet stationary ergodic process. Let $\left\{Y_{i}\right\}$ and $\left\{\tilde{Y}_{i}\right\}$ denote the process $\left\{X_{i}\right\}$ corrupted by the additive noise $\left\{N_{i}\right\}$, and $\left\{\tilde{N}_{i}\right\}$, respectively. Further let $\left\{Z_{i}\right\}$ denote the pattern process associated with $\left\{Y_{i}\right\}$. If $\left|S_{N}\right|<\infty$, then

$$
H(\mathbf{Z})=H(\tilde{\mathbf{Y}})
$$

It is interesting to note that the result of Proposition [3 can be rephrased to look more like those of Theorem 1 and Proposition [2. This is accomplished by observing that the process $\left\{\tilde{Y}_{i}\right\}$, used in Proposition [3, can also be constructed by

$$
\tilde{Y}_{i}= \begin{cases}Y_{i} & \text { if } Y_{i} \in S_{Y} \\ n_{o} & \text { otherwise }\end{cases}
$$

for an arbitrary $n_{o} \notin S_{Y}$. This is the construction of $\left\{\tilde{X}_{i}\right\}$ used in both Theorem 1 and Proposition 3 .

Proof of Proposition 3 ;

If $P\left(N_{i} \in S_{N}\right)=1$ then $N_{i}=\tilde{N}_{i}$ and there is nothing to prove, so we will assume that $P\left(N_{i} \in S_{N}\right)<1$. Let $\left\{\tilde{Z}_{i}\right\}$ denote the pattern process associated with the process $\left\{\tilde{Y}_{i}\right\}$. Since $\left\{\tilde{Y}_{i}\right\}$ is a discrete stationary ergodic process with finite entropy, Theorem 3 gives

$$
H(\tilde{\mathbf{Z}})=H(\tilde{\mathbf{Y}}) .
$$

Hence to complete the proof of Proposition 3, we just need to show that

$$
H(\mathbf{Z})=H(\tilde{\mathbf{Z}}) .
$$

Define

$$
\hat{Z}(n)_{i}=\left\{\begin{array}{cc}
Z_{i} & \text { if } \exists j \in[1, n] \backslash i \text { s.t. } Z_{i}=Z_{j} \\
y_{o} & \text { otherwise }
\end{array}\right.
$$

for some arbitrary $y_{o} \notin S_{Y}$. Clearly $\hat{Z}(n)$ uniquely determines $Z^{n}$ and vice versa, so in particular,

$$
H\left(Z^{n}\right)=H(\hat{Z}(n)) \quad \forall n>0 .
$$


Define

$$
I_{n_{o}}=\inf \left\{i>0: N_{i} \in S_{N}^{c}\right\} .
$$

We also observe the following: if $\tilde{Z}_{i} \neq \tilde{Z}_{I_{n_{o}}}$ then $Y_{i}=\tilde{Y}_{i}$ and if $\tilde{Z}_{i}=\tilde{Z}_{I_{n_{o}}}$ then w.p. 1 $Y_{i} \neq Y_{j}$ for all $j \neq i$. Hence we can construct $\hat{Z}(n)$ from $\tilde{Z}^{n}$ and $I_{n_{o}}$ w.p. 1 . Therefore

$$
H\left(\tilde{Z}^{n}, I_{n_{o}}\right) \geq H(\hat{Z}(n)) \quad \forall n>0
$$

and consequently,

$$
H\left(\tilde{Z}^{n}\right)+H\left(I_{n_{o}}\right) \geq H(\hat{Z}(n)) \quad \forall n>0 .
$$

Since $I_{n_{o}}$ is the waiting time for the first appearance of an element from $S_{N}^{c}$ in the i.i.d. process $\left\{N_{i}\right\}$, it is geometrically distributed, and in particular has finite entropy. Therefore

$$
\lim _{n \rightarrow \infty} \frac{H\left(I_{n_{o}}\right)}{n}=0
$$

which combined with (111) and (12) gives

$$
H(\tilde{\mathbf{Z}}) \geq H(\mathbf{Z})
$$

Defining $C(n)_{i}=\mathbb{1}_{\left\{\left\{\hat{Z}(n)_{i}=y_{o}\right\} \cap\left\{Y_{i} \in S_{Y}\right\}\right\}}$, we make the following observations: w.p. $1 Y_{i}=\tilde{Y}_{i}$ iff $C(n)_{i}=1$ or $\hat{Z}(n)_{i} \neq y_{o}$ and $\tilde{Y}_{i}=n_{o}$ iff $C(n)_{i}=0$ and $\hat{Z}(n)_{i}=y_{o}$. From these observations we conclude that given $C(n)$ and $\hat{Z}(n)$ we can reconstruct $\tilde{Z}^{n}$ w.p. 1 for all $n>0$. Hence, for all $n>0$,

$$
\begin{aligned}
H\left(\tilde{Z}^{n}\right) & \leq H(\hat{Z}(n), C(n)) \\
& \leq H(\hat{Z}(n))+H(C(n)) \\
& \stackrel{(a)}{=} H\left(Z^{n}\right)+H(C(n)) \\
& \leq H\left(Z^{n}\right)+\sum_{i=1}^{n} H\left(C(n)_{i}\right),
\end{aligned}
$$

where $(a)$ comes from (111).

Let

$$
P e_{i}^{(n)}=\operatorname{Pr}\left\{Y_{i} \in S_{Y}, Y_{j} \neq Y_{i} \forall j \in[1, n] \backslash i\right\} .
$$

Then

$$
\begin{aligned}
P e_{i}^{(n)} & =\operatorname{Pr}\left\{Y_{i} \in S_{Y}\right\} \operatorname{Pr}\left\{Y_{j} \neq Y_{i} \forall j \in[1, n] \backslash i \mid Y_{i} \in S_{Y}\right\} \\
& =\operatorname{Pr}\left\{Y_{i} \in S_{Y}\right\} \sum_{y \in S_{Y}} \operatorname{Pr}\left\{Y_{j} \neq y \forall j \in[1, n] \backslash i \mid Y_{i}=y\right\} \operatorname{Pr}\left\{Y_{i}=y \mid Y_{i} \in S_{Y}\right\} \\
& \leq \operatorname{Pr}\left\{Y_{i} \in S_{Y}\right\} \sum_{y \in S_{Y}} \operatorname{Pr}\left\{Y_{j} \neq y \forall j \in[1, n] \backslash i \mid Y_{i}=y\right\} .
\end{aligned}
$$


Without loss of generality assume that $i<n / 2$

$$
\begin{aligned}
P e_{i}^{(n)} & \leq \operatorname{Pr}\left\{Y_{i} \in S_{Y}\right\} \sum_{y \in S_{Y}} \operatorname{Pr}\left\{Y_{j} \neq y \forall j \in[i+1, i+n / 2-1] \mid Y_{i}=y\right\} \\
& \stackrel{(a)}{\leq} \operatorname{Pr}\left\{Y_{1} \in S_{Y}\right\} \sum_{y \in S_{Y}} \operatorname{Pr}\left\{Y_{j} \neq y \forall j \in[2, n / 2] \mid Y_{1}=y\right\}
\end{aligned}
$$

where $(a)$ follows from the stationarity of $Y$. Let

$$
P e^{(n)}=\operatorname{Pr}\left\{Y_{1} \in S_{Y}\right\} \sum_{y \in S_{Y}} \operatorname{Pr}\left\{Y_{j} \neq y \forall j \in[2, n / 2] \mid Y_{1}=y\right\} .
$$

Therefore we have

$$
P e^{(n)} \geq P e_{i}^{(n)} \quad \forall i
$$

By ergodicity we have

$$
\lim _{n \rightarrow \infty} \operatorname{Pr}\left\{Y_{j} \neq y \forall j \in[2, n / 2] \mid Y_{1}=y\right\}=0 \quad \forall y \in S_{Y}
$$

and since $\left|S_{Y}\right|<\infty$, (15) gives us $\lim _{n \rightarrow \infty} P e^{(n)}=0$. Hence there exists an $N$ such that $P e^{(n)}<1 / 2$ for all $n>N$ and (16) implies that

$$
H_{B}\left(P e_{i}^{(n)}\right) \leq H_{B}\left(P e^{(n)}\right) \quad \forall n \geq N,
$$

where $H_{B}$ is the binary entropy function. Substituting $P e_{i}^{(n)}$ into (14) and noting that $\mathbb{E}\left(C(n)_{i}\right)=P e_{i}^{(n)}$ gives

$$
\frac{H\left(\tilde{Z}^{n}\right)}{n} \leq \frac{H\left(Z^{n}\right)}{n}+\frac{1}{n} \sum_{i=1}^{n} H_{B}\left(P e_{i}^{(n)}\right) \quad \forall n \geq 0
$$

which combined with (17) gives us

$$
\frac{H\left(\tilde{Z}^{n}\right)}{n} \leq \frac{H\left(Z^{n}\right)}{n}+H_{B}\left(P e^{(n)}\right) \quad \forall n \geq N .
$$

Since $\lim _{n \rightarrow \infty} P e^{(n)}=0$,

$$
H(\tilde{\mathbf{Z}}) \leq H(\mathbf{Z})
$$

Combining (13) and (18) completes the proof.

Note that in the case where $\left\{N_{i}\right\}$ is a discrete i.i.d. process, Proposition 3 agrees with Theorem 3, In the case where $N_{i}$ has no point masses then, as Example 3 would suggest, Proposition 3 gives $H(\mathbf{Z})=0$.

Having verified that Proposition 3 is in agreement with previous results, let us examine a case where previous theorems do not apply. 
Example 6 Let $\left\{X_{i}\right\}$ be a first order Markov process on the set $\{1,2\}$ and let $\left\{N_{i}\right\}$ be i.i.d., independent of $\left\{X_{i}\right\}$, distributed according to the density

$$
f_{N}(n)=\frac{1}{\sqrt{8 \pi}} e^{-n^{2} / 2}+\frac{1}{2} \delta_{0}(n)
$$

where $\delta_{0}$ denotes a unit mass on 0 . Further let $Y_{i}=X_{i}+N_{i}$ and $\left\{Z_{i}\right\}$ be its associated pattern process. Since $\left\{Y_{i}\right\}$ is a hidden Markov process with memory on a continuous alphabet, previous results fail to capture the relationship between $H(\mathbf{Y})$ and $H(\mathbf{Z})$. However, Proposition 3 gives

$$
H(\mathbf{Z})=H(\tilde{\mathbf{Y}}),
$$

where $\tilde{Y}_{i}$ is the ternary hidden Markov process given by $X_{i}$ with probability 1/2 and an arbitrary $n_{o} \notin\{1,2\}$ with probability 1/2. We can also use Proposition 3 to lower bound $H(\mathbf{Z})$ in terms of $H(\mathbf{X})$. Noting that $\left\{\tilde{Y}_{i}\right\}$ is simply $\left\{X_{i}\right\}$ with erasures, we let $I_{i}$ denote the event of erasure at time $i$. Then

$$
\begin{aligned}
H\left(\tilde{Y}_{n} \mid \tilde{Y}^{n-1}\right) & \stackrel{(a)}{=} H\left(\tilde{Y}_{n}, I_{n} \mid \tilde{Y}^{n-1}\right) \\
& =H\left(I_{n}\right)+H\left(\tilde{Y}_{n} \mid \tilde{Y}^{n-1}, I_{n}\right) \\
& \stackrel{(b)}{=} H\left(I_{n}\right)+\operatorname{Pr}\left\{I_{n}=0\right\} H\left(X_{n} \mid \tilde{Y}^{n-1}, I_{n}=0\right)+0 \\
& \stackrel{(c)}{\geq} H\left(I_{n}\right)+\operatorname{Pr}\left\{I_{n}=0\right\} H\left(X_{n} \mid X^{n-1}, I_{n}=0\right) \\
& \stackrel{(d)}{=} 1+\frac{1}{2} H\left(X_{n} \mid X^{n-1}\right)
\end{aligned}
$$

where (a) follows from the fact that given $\tilde{Y}_{i}$ we know $I_{i}$, (b) from the fact that given $I_{i}=1, \tilde{Y}_{i}$ is a constant and given $I_{i}=0, \tilde{Y}_{i}=X_{i},(c)$ from a combination of the fact that $X_{n}$ is independent of $I^{n}$ and that conditioning decreases entropy, and finally, $(d)$ follows from the fact that $\left\{I_{i}\right\}$ is an i.i.d. Bernoulli $1 / 2$ process, independent of the process $\left\{X_{i}\right\}$. Combining (19) and (20) we get

$$
H(\mathbf{Z}) \geq \frac{1}{2} H(\mathbf{X})+1
$$

Note that (21) holds with equality when $\left\{X_{i}\right\}$ is i.i.d., as is readily seen to be implied by Theorem 1 .

\section{Stationary Ergodic Processes over Uncountable Alphabets}

Through the results of Proposition [2] and Proposition 3] we have seen two separate families of processes with memory on uncountable alphabets that share similar entropy rate properties. However, we are not able to extend such a relationship to the general stationary ergodic process. An interesting question that arises is what characteristics do the Markov processes of Proposition 2 and the additive noise processes of Proposition 3 share that allow for this characterization of the relationship 
between process and pattern entropy rates? In order to help answer this question, we examine the following Markov example which does not satisfy the requirements of Proposition 2

Example 7 Let $\left\{X_{i}\right\}$ be a first order Markov process on $[0,1]$ with a uniform stationary distribution. Furthermore, conditioned on $X_{i}, X_{i+1}=X_{i}$ with probability $1 / 2$ and $X_{i+1}$ is drawn uniformly on $[0,1]$ with probability $1 / 2$. It is easy to see that $\left\{X_{i}\right\}$ does not satisfy the conditions of Proposition 2 . In this case, $S=\emptyset$ and therefore the sequence $\left\{\tilde{X}_{i}\right\}$ is constant and

$$
H(\tilde{\mathbf{X}})=0
$$

We also observe that at any time $i+1$ we either see a new symbol with probability $1 / 2$ or we repeat $X_{i}$ with probability $1 / 2$. Therefore,

$$
H(\mathbf{Z})=1
$$

not $H(\tilde{\mathbf{X}})$ as would be assumed from the relationship between pattern and process entropy rates found in Proposition 2 and Proposition [3. Hence, unlike the processes described in Proposition 2 and Proposition [3, we see that

$$
H(\mathbf{Z}) \neq H(\tilde{\mathbf{X}}) .
$$

Example 7 suggests that one of the important characteristics shared by the processes in Proposition [2] and Proposition 3, which allow for the equality between $H(\mathbf{Z})$ and $H(\tilde{\mathbf{X}})$, is the control over the repeatability of density points. In other words, assuring that for the most part only elements in $S$ are likely to be seen more than once. This characteristic is also demonstrated by the i.i.d. processes of Theorem 1 , which share the equality between $H(\mathbf{Z})$ and $H(\tilde{\mathbf{X}})$.

With this in mind we can try to extend this characteristic to general stationary ergodic processes in hopes of developing a similar entropy rate relation. Before we state the next theorem, let us define some notation and make rigorous the criterion of repeatability described above. Given a stationary ergodic process $\left\{X_{i}\right\}$ on $\mathbb{R}$, let $S=\left\{x \in \mathbb{R}: \operatorname{Pr}\left\{X_{1}=x\right\}>0\right\}$ and $R=\left\{x \in \mathbb{R}: \operatorname{Pr}\left\{\exists j \geq 2: X_{j}=X_{1} \mid X_{1}=\right.\right.$ $x\}>0\}$. Let $S=\left\{s_{1}, s_{2}, \ldots, s_{|S|}\right\}$ and $P_{i}=\operatorname{Pr}\left\{X_{1}=s_{i}\right\}$. Without loss of generality we will assume that the elements of $S$ are ordered such that $P_{1} \geq P_{2} \geq \ldots$

Theorem 4 Let $\left\{X_{i}\right\}$ be a stationary ergodic process on $\mathbb{R}$ with $\operatorname{Pr}\left\{X_{1} \in R\right\}=$ $\operatorname{Pr}\left\{X_{1} \in S\right\}$. Let $\left\{Z_{i}\right\}$ be the associated pattern process. Define the process

$$
\tilde{X}_{i}= \begin{cases}X_{i} & \text { if } X_{i} \in S \\ x_{o} & \text { otherwise }\end{cases}
$$

for some arbitrary $x_{o} \notin S$. If $|S|<\infty$, then

$$
H(\mathbf{Z})=H(\tilde{\mathbf{X}})
$$


Otherwise, if $|S|$ is infinite and there exists $\beta>2$ such that

$$
\lim _{i \rightarrow \infty} \frac{P_{i}}{(1 / i)^{\beta}}=0
$$

then

$$
H(\mathbf{Z})=H(\tilde{\mathbf{X}})
$$

It should be to noted that both Proposition [2] and Proposition 3 are special cases of Theorem 4.

The requirement $\operatorname{Pr}\left\{X_{1} \in R\right\}=\operatorname{Pr}\left\{X_{1} \in S\right\}$, is the mathematical equivalent of the statement that only elements in $S$ are likely to be seen more than once. While the $\beta$-convergence requirement is a technicality needed in the proof, which may prove to be non-essential.

Hence we see that controlling repeatability of density points is, essentially, a sufficient condition for establishing equality between $H(\mathbf{Z})$ and $H(\tilde{\mathbf{X}})$. Furthermore, Example 7 suggests that it is a necessary condition. Hence, the $\beta$-convergence requirement aside, there is reason to believe that Theorem 4 in some sense describes the largest family of stationary ergodic processes over uncountable alphabets for which the equality between $H(\mathbf{Z})$ and $H(\tilde{\mathbf{X}})$ holds.

Proof of Theorem 4:

We can assume that $\operatorname{Pr}\left\{X_{1} \in S\right\}<1$, otherwise there is nothing to prove. If $|S|<\infty$, then $H\left(\tilde{X}_{1}\right)<\infty$. In the case where $|S|$ is infinite, then the fact that $\beta>2$ and

$$
\lim _{i \rightarrow \infty} \frac{P_{i}}{(1 / i)^{\beta}}=0
$$

implies that $H\left(\tilde{X}_{1}\right)<\infty$. Since $\left\{\tilde{X}_{i}\right\}$ is a discrete stationary ergodic process with finite entropy, Theorem $[3$ gives

$$
H(\tilde{\mathbf{X}})=H(\tilde{\mathbf{Z}})
$$

where $\left\{\tilde{Z}_{i}\right\}$ is the associated pattern process. To complete the proof of Theorem 4, we need to show that

$$
H(\mathbf{Z})=H(\tilde{\mathbf{Z}}) .
$$

For $x \in S$ define $I_{x}=\inf \left\{i>0: X_{i}=x\right\}$ and $I_{x}^{(n)}=I_{x} \mathbb{1}_{\left\{I_{x} \leq n\right\}}-\mathbb{1}_{\left\{I_{x}>n\right\}}$. Hence $I_{x}^{(n)}$ has an alphabet of size $n+1$ and therefore

$$
H\left(I_{x}^{(n)}\right) \leq \log (n+1)
$$

Given $B \subseteq S$ such that $|B|<\infty$, let $P_{B}=\operatorname{Pr}\left\{X_{1} \in S \cap B^{c}\right\}$ and $C_{B}^{(n)}=$ $\mathbb{1}_{\left\{X_{1}, X_{2}, \ldots, X_{n} \notin S \cap B^{c}\right\}}$. 
If $C_{B}^{(n)}=1$ then given $\left\{I_{x}^{(n)}\right\}_{x \in B}$, we know all the labels of the elements of $S$ which appear in $X^{n}$. Hence for $n \geq 1$ and conditioned on $C_{B}^{(n)}=1$, given $Z^{n}$ and $\left\{I_{x}^{(n)}\right\}_{x \in B}$ we can reconstruct $\tilde{Z}^{n}$. Therefore

$$
\begin{aligned}
H\left(\tilde{Z}^{n} \mid C_{B}^{(n)}=1\right) & \leq H\left(Z^{n} \mid C_{B}^{(n)}=1\right)+H\left(\left\{I_{x}^{(n)}\right\}_{x \in B} \mid C_{B}^{(n)}=1\right) \\
& \leq H\left(Z^{n}\right)+H\left(\left\{I_{x}^{(n)}\right\}_{x \in B}\right) \\
& \leq H\left(Z^{n}\right)+|B| \log (n+1) \quad \forall n \geq 1 .
\end{aligned}
$$

Given $i$, we now wish to examine the probability

$$
\begin{aligned}
\operatorname{Pr}\left\{\exists j>i: X_{j}=X_{i} \mid X_{i} \notin S\right\} & \stackrel{(a)}{=} \operatorname{Pr}\left\{\exists j \geq 2: X_{j}=X_{1} \mid X_{1} \notin S\right\} \\
& \stackrel{(b)}{\leq} \operatorname{Pr}\left\{\exists j \geq 2: X_{j}=X_{1} \mid X_{1} \notin R\right\} \\
& \leq \sum_{j=2}^{\infty} \operatorname{Pr}\left\{X_{j}=X_{1} \mid X_{1} \notin R\right\}
\end{aligned}
$$

where $(a)$ follows from the stationarity of the process $\left\{X_{i}\right\}$ and $(b)$ from the fact that $S \subseteq R$ and $\operatorname{Pr}\left\{X_{1} \in R\right\}=\operatorname{Pr}\left\{X_{1} \in S\right\}$ implies that $\operatorname{Pr}\left\{X_{1} \in R \cap S^{c}\right\}=0$.

Let $f_{j}\left(x_{1}, x_{j}\right)$ be the distribution on $\left(X_{1}, X_{j}\right)$ given $X_{1} \notin R$. Therefore

$$
\operatorname{Pr}\left\{X_{j}=X_{1} \mid X_{1} \notin R\right\}=\int_{x_{1} \in R^{c}} \int_{x_{j}=x_{1}} f_{j}\left(x_{1}, x_{j}\right) d x_{j} d x_{1} .
$$

Assume that $\operatorname{Pr}\left\{X_{j}=X_{1} \mid X_{1} \notin R\right\}>0$, then (26) implies that there exists $x_{1} \in R^{c}$ such that

$$
\int_{x_{j}=x_{1}} f_{j}\left(x_{1}, x_{j}\right) d x_{j}>0 .
$$

This is only possible if $\operatorname{Pr}\left\{X_{1}=X_{j}=x_{1} \mid X_{1} \notin R\right\}>0$. Therefore $\operatorname{Pr}\left\{X_{j}=X_{1} \mid X_{1}=\right.$ $\left.x_{1}\right\}>0$ and by definition of $R, x_{1} \in R$. This is a contradiction since $x_{1} \in R^{c}$. Hence

$$
\operatorname{Pr}\left\{X_{j}=X_{1} \mid X_{1} \notin R\right\}=0 \quad \forall j \geq 2
$$

and (25) gives

$$
\operatorname{Pr}\left\{\exists j>i: X_{j}=X_{i} \mid X_{i} \notin S\right\}=0 .
$$

Therefore w.p. 1, only elements in $S$ will appear more than once. Hence conditioned on $C_{B}^{(n)}=1$, given $\left\{I_{x}^{(n)}\right\}_{x \in B}$ we know the labels of all the elements in $X^{n}$ except those that appear at most once w.p. 1 . Therefore for $n \geq 1$ and conditioned on $C_{B}^{(n)}=1$, given $\left\{I_{x}^{(n)}\right\}_{x \in B}$ and $\tilde{Z}^{n}$, we can reconstruct $Z^{n}$ w.p. 1 . Hence

$$
\begin{aligned}
H\left(Z^{n} \mid C_{B}^{(n)}=1\right) & \leq H\left(\tilde{Z}^{n} \mid C_{B}^{(n)}=1\right)+H\left(\left\{I_{x}^{(n)}\right\}_{x \in B} \mid C_{B}^{(n)}=1\right) \\
& \leq H\left(\tilde{Z}^{n}\right)+H\left(\left\{I_{x}^{(n)}\right\}_{x \in B}\right) \\
& \leq H\left(\tilde{Z}^{n}\right)+|B| \log (n+1) \quad \forall n \geq 1 .
\end{aligned}
$$


If $|S|<\infty$, then set $B=S$. Therefore $P_{B}=0, C_{B}^{(n)}=1$ w.p. 1 and equations (24) and (28) give

$$
\begin{array}{ll}
H\left(Z^{n}\right) \leq H\left(\tilde{Z}^{n}\right)+|S| \log (n+1) & \forall n \geq 1 \\
H\left(\tilde{Z}^{n}\right) \leq H\left(Z^{n}\right)+|S| \log (n+1) & \forall n \geq 1 .
\end{array}
$$

The finiteness of $|S|$ then implies

$$
H(\mathbf{Z})=H(\tilde{\mathbf{Z}}) .
$$

To complete the proof of Theorem 4 we need to address the case where $|S|$ is infinite. Choose $\gamma \in(0,1)$ such that $\alpha \triangleq \gamma(\beta-1)>1$. Such a $\gamma$ can be found since $\beta>2$. Let $m(n)=\left\lceil n^{\gamma}\right\rceil$. Since

$$
\lim _{i \rightarrow \infty} \frac{P_{i}}{(1 / i)^{\beta}}=0
$$

and $m(n)$ is an unbounded increasing sequence, there exists $N>0$ such that

$$
m(n)^{\beta} P_{m(n)}<1 \quad \forall n>N .
$$

Construct a sequence of sets $B_{n} \subseteq S$ as follows, $B_{n}=\left\{s_{1}, \ldots, s_{m(n)}\right\}$ for all $n>N$. Therefore

$$
\begin{aligned}
P_{B_{n}} & =\operatorname{Pr}\left\{X_{1} \in S \cap B_{n}^{c}\right\} \\
& \leq \sum_{i=m(n)+1}^{\infty} \operatorname{Pr}\left\{X_{1}=s_{i}\right\} \\
& =\sum_{i=m(n)+1}^{\infty} P_{i} \\
& \stackrel{(a)}{\leq} \sum_{i=m(n)+1}^{\infty} \frac{1}{i^{\beta}} \\
& \leq \int_{m(n)}^{\infty} \frac{1}{x^{\beta}} d x \\
& =\frac{1}{\beta-1}(m(n))^{1-\beta} \\
& =\frac{1}{\beta-1}\left\lceil n^{\gamma} 1^{1-\beta}\right. \\
& \stackrel{(b)}{\leq} \frac{1}{\beta-1} n^{\gamma(1-\beta)} \\
& =\frac{1}{\beta-1} n^{-\alpha} \quad \forall n>N,
\end{aligned}
$$


where $(a)$ follows from the fact that $n>N$ and $(b)$ from the fact that $\beta>2$. Therefore

$$
\begin{aligned}
H\left(\tilde{Z}^{n}\right) & \leq H\left(\tilde{Z}^{n}, C_{B_{n}}^{(n)}\right) \\
& =H\left(C_{B_{n}}^{(n)}\right)+H\left(\tilde{Z}^{n} \mid C_{B_{n}}^{(n)}\right) \\
& \leq 1+\operatorname{Pr}\left\{C_{B_{n}}^{(n)}=1\right\} H\left(\tilde{Z}^{n} \mid C_{B_{n}}^{(n)}=1\right)+\operatorname{Pr}\left\{C_{B_{n}}^{(n)}=0\right\} H\left(\tilde{Z}^{n} \mid C_{B_{n}}^{(n)}=0\right) \\
& \leq 1+H\left(\tilde{Z}^{n} \mid C_{B_{n}}^{(n)}=1\right)+n P_{B_{n}} H\left(\tilde{Z}^{n} \mid C_{B_{n}}^{(n)}=0\right) \\
& \leq 1+H\left(\tilde{Z}^{n} \mid C_{B_{n}}^{(n)}=1\right)+n P_{B_{n}} H\left(\tilde{Z}^{n}\right) \\
& \leq 1+H\left(\tilde{Z}^{n} \mid C_{B_{n}}^{(n)}=1\right)+n^{2} P_{B_{n}} \log n \\
& \stackrel{(b)}{\leq} 1+H\left(Z^{n}\right)+\left|B_{n}\right| \log (n+1)+n^{2} P_{B_{n}} \log n \\
& =1+H\left(Z^{n}\right)+m(n) \log (n+1)+n^{2} P_{B_{n}} \log n \\
& (c) \\
& \leq 1+H\left(Z^{n}\right)+\left(n^{\gamma}+1\right) \log (n+1)+n^{2-\alpha} \log n \quad \forall n>N,
\end{aligned}
$$

where $(a)$ follows from the fact that $\tilde{Z}_{i}$ has an alphabet of at most $i,(b)$ follows from (24), and $(c)$ from (29). Similarly using (28) we get

$$
H\left(Z^{n}\right) \leq 1+H\left(\tilde{Z}^{n}\right)+\left(n^{\gamma}+1\right) \log (n+1)+n^{2-\alpha} \log n \quad \forall n>N .
$$

Since $\gamma \in(0,1)$ and $\alpha>1,(30)$ and (31) give

$$
H(\mathbf{Z})=H(\tilde{\mathbf{Z}})
$$

completing the proof of Theorem 4 .

\section{Growth Rates}

Now that we have explored the relationship between the entropy rate of the original process and the associated pattern process, we turn our attention to possible growth rates for the block entropy of a pattern sequence. In other words, having looked at the limit, we now look at the asymptotic growth rates.

Theorem 5 For any $\delta>0$ there exists an i.i.d. process $\left\{X_{i}\right\}$ such that its associated pattern sequence satisfies

$$
\lim _{n \rightarrow \infty} \frac{H\left(Z_{n+1} \mid Z^{n}\right)}{(\ln n)^{1-\delta}}=\infty
$$

Note that since $Z_{n+1}$ lies in an alphabet of size at most $n+1$ we have, for any process, not even necessarily stationary,

$$
\limsup _{n \rightarrow \infty} \frac{H\left(Z_{n+1} \mid Z^{n}\right)}{\log n} \leq 1 .
$$


Theorem 5 then says that the growth rate $\log n$ is essentially, up to a factor which is sub-polynomial in $\log n$, achievable by an i.i.d. process.

We dedicate the remainder of this section to the proof of Theorem [5. Let $X_{i}$ be i.i.d. $\sim f_{X}$, where $X_{i}$ takes values in an arbitrary space $\mathcal{A}$, and $\left\{Z_{i}\right\}$ be the associated pattern sequence. Define $S=\left\{x \in \mathcal{A}: \operatorname{Pr}\left\{X_{1}=x\right\}>0\right\}$.

Claim $4 H\left(\Phi_{B}\left[f_{X}\right]\right)$ is increasing in $B$, i.e., for any $B_{1} \subseteq B_{2} \subseteq S$

$$
H\left(\Phi_{B_{1}}\left[f_{X}\right]\right) \leq H\left(\Phi_{B_{2}}\left[f_{X}\right]\right)
$$

Proof of Claim 4 .

This is nothing but a data-processing inequality. Indeed, let $Y \sim \Phi_{B_{2}}\left[f_{X}\right]$ and let

$$
U= \begin{cases}Y & \text { if } Y \in B_{1} \\ x_{o} & \text { otherwise. }\end{cases}
$$

Clearly $U \sim \Phi_{B_{1}}\left[f_{X}\right]$ and $U$ is a deterministic function of $Y$, thus the claim follows.

Proposition 4 For any $B \subseteq S$

$$
H\left(Z_{n+1} \mid Z^{n}\right) \geq H\left(\Phi_{B}\left[f_{X}\right]\right)\left[1-|B| \exp \left(-n \min _{b \in B} \operatorname{Pr}\{X=b\}\right)\right]
$$

Proof of Proposition 4

Letting $P_{X}^{n}$ denote the distribution of $X^{n}$, for any $B \subseteq S$,

$$
\begin{aligned}
H\left(Z_{n+1} \mid Z^{n}\right) & \geq H\left(Z_{n+1} \mid X^{n}\right) \\
& =\int_{\mathcal{A}^{n}} H\left(Z_{n+1} \mid X^{n}=x^{n}\right) d P_{X}^{n}\left(x^{n}\right) \\
& =\int_{\mathcal{A}^{n}} H\left(\Phi_{A\left(x^{n}\right)}\left[f_{X}\right]\right) d P_{X}^{n}\left(x^{n}\right) \\
& \geq \int_{\left\{x^{n}: B \subseteq A\left(x^{n}\right)\right\}} H\left(\Phi_{A\left(x^{n}\right)}\left[f_{X}\right]\right) d P_{X}^{n}\left(x^{n}\right) \\
& \geq H\left(\Phi_{B}\left[f_{X}\right]\right) \operatorname{Pr}\left\{B \subseteq A\left(X^{n}\right)\right\},
\end{aligned}
$$

where the last inequality follows from the monotonicity property in Claim 4 and $A\left(X^{n}\right)$ defined to be the set of distinct elements in $\left\{X_{1}, \ldots, X_{n}\right\}$. Now, for any $B \subseteq S$,

$$
\begin{aligned}
\operatorname{Pr}\left(B \nsubseteq A\left(X^{n}\right)\right) & =\operatorname{Pr}\left(\bigcup_{b \in B}\left\{b \notin A\left(X^{n}\right)\right\}\right) \\
& \leq \sum_{b \in B} \operatorname{Pr}\left\{b \notin A\left(X^{n}\right)\right\}
\end{aligned}
$$




$$
\begin{aligned}
& =\sum_{b \in B}(1-\operatorname{Pr}\{X=b)\}^{n} \\
& \leq|B|\left(1-\min _{b \in B} \operatorname{Pr}\{X=b\}\right)^{n} \\
& \leq|B| \exp \left(-n \min _{b \in B} \operatorname{Pr}\{X=b\}\right) .
\end{aligned}
$$

The proposition now follows by combining (33) with (34).

Besides being used in the proof of Theorem 5, Proposition 4 also gives the following corollary which will be used in the proof of Theorem [1.

\section{Corollary 6}

$$
\liminf _{n \rightarrow \infty} H\left(Z_{n+1} \mid Z^{n}\right) \geq H\left(\Phi_{S}\left[f_{X}\right]\right),
$$

regardless of the finiteness of the right side of the inequality.

Proof of Corollary 6 .

Take a sequence $\left\{B_{k}\right\}$ of finite subsets $B_{k} \subseteq S$ satisfying

$$
\lim _{k \rightarrow \infty} H\left(\Phi_{B_{k}}\left[f_{X}\right]\right)=H\left(\Phi_{S}\left[f_{X}\right]\right) .
$$

Proposition 4 implies, for each $k$,

$$
\liminf _{n \rightarrow \infty} H\left(Z_{n+1} \mid Z^{n}\right) \geq H\left(\Phi_{B_{k}}\left[f_{X}\right]\right)
$$

completing the proof by taking $k \rightarrow \infty$ on the right side of (35).

Proof of Theorem 5:

Consider the case where $\left\{X_{i}\right\}$ are generated i.i.d. $\sim P$, where $P$ is a distribution on $\mathbb{N}$ and $p_{j}=P\left(X_{1}=j\right)$ is a non-increasing sequence. Letting $D_{l}=\sum_{i=1}^{l} p_{i} \log \frac{1}{p_{i}}$ it follows by taking $B=B_{l}=\{1, \ldots, l\}$ in Proposition 4 that

$$
\begin{aligned}
H\left(Z_{n+1} \mid Z^{n}\right) & \geq H\left(\Phi_{B_{l}}\left[f_{X}\right]\right)\left[1-\left|B_{l}\right| \exp \left(-n \min _{b \in B_{l}} \operatorname{Pr}(X=b)\right)\right] \\
& \geq D_{l}\left[1-l \exp \left(-n p_{l}\right)\right]
\end{aligned}
$$

implying, by the arbitrariness of $l$,

$$
H\left(Z_{n+1} \mid Z^{n}\right) \geq \max _{l} D_{l}\left[1-l \exp \left(-n p_{l}\right)\right] .
$$

Consider now the distribution

$$
p_{i}=P\left(X_{1}=i\right)=\frac{c(\varepsilon)}{i(\ln i)^{1+\varepsilon}},
$$


for some $\varepsilon>0$, where $c(\varepsilon)$ is the normalization constant. In this case

$$
D_{l}=\sum_{i=1}^{l} \frac{c(\varepsilon)}{i(\ln i)^{1+\varepsilon}} \log \frac{i(\ln i)^{1+\varepsilon}}{c(\varepsilon)} \sim \sum_{i=1}^{l} \frac{1}{i(\ln i)^{\varepsilon}} \sim(\ln l)^{1-\varepsilon}
$$

thus (36) implies, taking $l \approx n^{(1-\varepsilon) /(1+\varepsilon)}$,

$$
\begin{aligned}
H\left(Z_{n+1} \mid Z^{n}\right) & \geq D_{l}\left[1-l \exp \left(-n p_{l}\right)\right] \\
& \sim(\ln l)^{1-\varepsilon}\left[1-l \exp \left(-n p_{l}\right)\right] \\
& \sim(\ln l)^{1-\varepsilon}\left[1-l \exp \left(-n / l^{1+\varepsilon}\right)\right] \\
& \sim(\ln n)^{1-\varepsilon}\left[1-n \exp \left(-n^{\varepsilon}\right)\right] \\
& \sim(\ln n)^{1-\varepsilon} .
\end{aligned}
$$

Thus (32) is satisfied under the distribution in (37) with any $\varepsilon<\delta$.

\section{Conclusion}

We have characterized the relationship between the entropy rate of a source and that of its pattern process for i.i.d., discrete Markov, discrete stationary ergodic, and a broad family of uncountable alphabet stationary ergodic processes. Besides determining the fundamental compression limits for a pattern sequence, the relationship between pattern and process entropy rate helps to quantify how much of the total information contained in the original stochastic process is encompassed in its pattern sequence. For the case where the pattern entropy rate is infinite, we characterized achievable growth rates for the block entropy of a pattern sequence.

\section{A Proof of Theorem 1}

If $|S|=0$, then $\operatorname{Pr}\left\{\exists i \neq j: X_{i}=X_{j}\right\}=0$. Therefore $H\left(Z^{n}\right)=0$ for all $n$. This implies that $H(\mathbf{Z})=0$ which agrees with Theorem 1 Hence we just need to prove Theorem 1 for the case where $|S|>0$.

Note that Corollary [6] and the fact that regardless of the finiteness of $H\left(X_{1}\right)$, $H\left(Z_{1}\right)<\infty$ and $H\left(Z_{n} \mid Z^{n-1}\right)<\infty$ for all $n$ gives

$$
\liminf _{n \rightarrow \infty} \frac{H\left(Z^{n}\right)}{n} \geq H\left(\tilde{X}_{1}\right) .
$$

For the reverse inequality, look at

$$
\begin{aligned}
\limsup _{n \rightarrow \infty} \frac{H\left(Z^{n}\right)}{n} & \stackrel{(a)}{\leq} \limsup _{n \rightarrow \infty} \frac{H\left(\tilde{X}^{n}\right)}{n} \\
& \stackrel{(b)}{=} H\left(\tilde{X}_{1}\right),
\end{aligned}
$$


where $(a)$ comes from the fact that given $\tilde{X}^{n}$ we can reconstruct $Z^{n}$ w.p. 1 and $(b)$ from the fact that $\left\{\tilde{X}_{i}\right\}$ is and i.i.d. process. Combining (38) and (39) and noting that $\left\{\tilde{X}_{i}\right\}$ is an i.i.d. process completes the proof of Theorem 1 .

\section{References}

[1] K. L. Chung, A note on the ergodic theorem of information theory, Ann. Math. Stat., 32, 1961, p. 612-614.

[2] G. Gemelos, T. Weissman, On the Entropy Rate of Pattern Processes, Hewlett Packard Tech Report HPL-2004-159, September 2004.

[3] G. Gemelos, T. Weissman, On the entropy rate of pattern processes, Proceedings of the 2005 Data Compression Conference, Snowbird, Utah, March 2005.

[4] N. Jevtić, A. Orlitsky, N. Santhanam, Universal compression of unknown alphabets, Proceedings of the 2002 IEEE International Symposium on Information Theory, p. 302, Lausanne, Switzerland, July 2002.

[5] A. Orlitsky, N. P. Santhanam, Performance of universal codes over infinite alphabets, Proceedings of the 2003 Data Compression Conference, Snowbird Utah, March 2003.

[6] A. Orlitsky, N. P. Santhanam, J. Zhang, Bounds on compression of unknown alphabets, Proceedings of the 2003 IEEE International Symposium on Information Theory, p. 111, Yokohama, Japan, June 2003.

[7] A. Orlitsky, N.P. Santhanam, K. Viswanathan, J. Zhang, Limit results on pattern entropy, Proceedings of the IEEE Information Theory Workshop, San Antonio, Texas, October 2004.

[8] A. Orlitsky, N.P. Santhanam, and J. Zhang, Universal compression of memoryless sources over unknown alphabets, IEEE Transactions on Information Theory, IT-50:7 (July 2004), p. 1469-1481.

[9] A. Orlitsky, N. P. Santhanam, K. Viswanathan, J. Zhang, Limit Results on Pattern Entropy, Submitted to IEEE Trans. on Information Theory.

[10] G. I. Shamir, On the MDL principle for universal compression of unknown alphabets, Proceedings of the 2002 Allerton Conference on Communication, Control, and Computing, p. 1238-1247, Allerton, IL, October 2004. 
[11] G. I. Shamir, Universal lossless compression with unknown alphabets - The average case, Proceedings of the 2004 IEEE International Symposium on Information Theory, p. 27, Chicago, IL, July 2004.

[12] G. I. Shamir, Sequence-patterns entropy and infinite alphabets, Proceedings of the 2004 Allerton Conference on Communication, Control and Computing, Allerton, IL, October 2004.

[13] G. I. Shamir, Sequential universal lossless techniques for compression of patterns and their description length, Proceedings of the 2004 Data Compression Conference, p. 419-428, Snowbird, Utah, March 2004.

[14] G. I. Shamir and L. Song, On the entropy of patterns of i.i.d. sequences, Proceedings of the 2003 Allerton Conference on Communication, Control, and Computing, Allerton, IL, October 2003. 